\title{
Etat de connaissance sur le paléoenvironnement des 20000 ans B.P. dans le delta de l'Ouémé au Bénin: influence des facteurs géologiques et paléoclimatiques
}

\author{
A. R. M. A. ADECHINA ${ }^{1 *}$, C. N. KELOME ${ }^{2}$, C. KAKI ${ }^{2}$, R. A. $\mathrm{LAIBI}^{2}$ et \\ J. B. HOUNKPE ${ }^{2}$
}

\author{
${ }^{1}$ ICMPA-UNESCO Chair, Université d'Abomey-Calavi, 072 BP 50 Cotonou, Bénin. \\ ${ }^{2}$ Département des Sciences de la Terre, Université d'Abomey-Calavi, 01 BP 526 Cotonou, Bénin. \\ *Auteur correspondant; E-mail : radechina@yahoo.fr
}

\section{RESUME}

Les écosystèmes naturels ne sont pas indifférents des fortes variabilités climatiques intersaisonnières de ces dernières années. L'évaluation des impacts actuels de ces variabilités nécessitent la connaissance des paléoenvironnements. Ce travail de synthèse fait l'état des connaissances sur l'évolution des environnements géologiques et paléoclimatiques des 20000 ans B.P. dans l'estuaire de l'Ouémé au Bénin. De façon spécifique, il s'agit, de caractériser l'environnement de dépôt de différentes séquences sédimentaires, d'analyser le contenu palynologique des sédiments et de situer le paléoclimat dépôts dans le contexte intertropical africain et global. L'approche utilisée est basée sur la collecte et la synthèse des coupes géologiques et palynologiques. Il ressort que depuis le dernier maximum glaciaire jusqu'à 14000 ans B.P., les forêts et les niveaux marins ont régressé. Une colonisation forestière a été notée entre 13400-12500 ans B.P., mais elle a été très tôt interrompue par le Younger Dryas. Dès l'Holocène (10300 ans B.P.) les forêts ont proliféré, l'estuaire de l'Ouémé a été transformé en ria. Il s'en est suivi vers 4200 ans B.P., une péjoration climatique au cours de laquelle l'influence anthropique a été exprimée. Il est envisagé une étude qui combine la sédimentologie et les espèces de dinoflagellés, un outil de reconstitution du paléoclimat.

(C) 2018 International Formulae Group. All rights reserved.

Mots clés : Paléoenvironnement, dernier maximum glaciaire, séquences sédimentaires, palynologie, estuaire de l'Ouémé.

\section{State of knowledge on the palaeoenvironment of the 20,000 years B.P. in the Oueme estuary in Benin: influence of geological and paleoclimatic factors}

\begin{abstract}
Natural ecosystems are not indifferent from the high inter-seasonal climate variability of recent years. Evaluation of the current impacts of these variabilities requires knowledge of the evolution of past environments. This work of synthesis shows the state of knowledge on the evolution of the geological and palaeoclimatic environments of the 20,000 years B.P. The aim was to specifically characterize the depositional environment of different sedimentary sequences, to analyze the palynological content of the sediments and to situate the paleoclimate deposits in the African and global intertropical context. The approach used is based on the collection and synthesis of geological and palynological sections. It brings out that since the last glacial
\end{abstract}


maximum till 14000 years B.P., forests and sea levels have regressed. We noticed forest colonization between 13400-12500 years B.P., but it was earlier interrupted by the Younger Dryas. Since the Holocene (10300 years B.P.) the forests have proliferated, the estuary of the Ouémé has been transformed into ria. This was followed by a pejorative climate around 4200 years B.P. in which the anthropogenic influence was expressed. A study is envisaged that combines the sedimentology and the species of dinoflagellates, a real tool of reconstitution of the paleoclimate.

(C) 2018 International Formulae Group. All rights reserved.

Keywords: Palaeoenvironment, last glacial maximum, sedimentary sequences, palynology, Oueme estuary

\section{INTRODUCTION}

Les milieux margino-littoraux représentent une interface entre la terre et l'océan, et regorgent d'importants écosystèmes capables d'enregistrer les changements environnementaux dus aux facteurs géologiques, climatiques, socio-économiques, etc. (Green et al., 2013). En terme climatique, les périodes de trois décennies ont été de plus en plus chaudes que les précédentes depuis 1850 (IPCC, 2014). Les conséquences de ces mutations récentes se manifestent par des irrégularités pluviométriques, la modification des températures de surface, l'effet de serre... (Guo et al., 2015). Le long des côtes du golfe de Guinée, le milieu margine-littoral regroupe les plaines littorales et leurs prolongements à l'intérieur du continent (Kaki et al., 2011). L'estuaire de l'Ouémé dans le domaine margino-littoral du Bénin n'est pas épargné de ces perturbations d'échelle planétaire. En effet, depuis trois décennies, il est frappé par la récession des précipitations, la hausse des températures maximales, la récurrence des inondations... (Zannou et Vodounou, 2011). $\mathrm{Du}$ point de vue géologique, la genèse de l'estuaire de l'Ouémé coïncide avec le dernier maximum glaciaire situé entre 21000-18000 ans B.P. (Mix et al, 2001; Agassounon, 2002). Mais, la plus intense phase de dépôt fut à la faveur de la transgression Holocène datée de 7500-5500 ans B.P. (Ballouche et al., 2001 ; Agassounon, 2002). Vers la fin de l'Holocène moyen (4000 ans B.P.), les sédiments déposés progradent en direction du complexe lagunaire «lac Nokoué-lagune de Porto-Novo» situé en aval (Kelome et al., 2012).

Pour l'ensemble des anciens travaux, la chronologie des évènements géologiques dans l'estuaire de l'Ouémé est basée sur les données géochronologiques disponibles dans le secteur de la Sô (un ancien bras déconnecté de l'Ouémé) ainsi que dans les plaines adjacentes. Il est noté une insuffisance remarquable de ces données, du côté de l'Ouémé, le principal bassin versant. En outre, dans le contexte de réchauffement climatique qui a marqué ces trois dernières décennies, la validation et la fiabilité des modèles de prédiction sur l'évolution future contraignent à s'appuyer sur une connaissance solide de l'histoire des environnements. Or, les sédiments du delta de l'Ouémé sont susceptibles d'enregistrer les messages des grands événements climatiques et géologiques passés. Le problème relevé des travaux antérieurs renseigne que; des séquences sédimentaires témoins des évènements globaux ont pu se déposer dans l'estuaire de l'Ouémé mais le contexte paléoclimatique n'est pas analyser. Ce travail de synthèse bibliographique vise à reconstituer le paléoenvironnement des dépôts de l'estuaire de l'Ouémé en combinant la palynologie et la sédimentologie. De façon spécifique, il s'agit d'analyser l'environnement de dépôt des séquences sédimentaires; de caractériser le contenu palynologique des sédiments; enfin de situer le paléoclimat des dépôts dans le contexte intertropical africain et global.

\section{METERIEL ET METHODES Site d'étude}

L'estuaire de l'Ouémé est localisé dans la partie méridionale du Bénin, un des pays Ouest-africains intertropicaux (Figure 1). Le fleuve Ouémé est le principal cours d'eau du réseau hydrographique dont les affluents sont l'Okpara, et le Zou. Cet estuaire est intégré au bassin sédimentaire côtier du Bénin, lequel est formé du domaine des 
plateaux et le domaine margino-littoral. Les formations géologiques sont constituées de dépôts quaternaires qui reposent sur une épaisse couche de grès, d'argiles, du calcaire... dont les âges remontent jusqu'au Cénomanien. La lithologie des dépôts quaternaires est composée d'argiles, de sables, de vases, de tourbe (Laibi, 2011). Le climat est du type subéquatorial avec des températures moyennes de l'aire d'environ $28,33{ }^{\circ} \mathrm{C}$ à la grande saison sèche et $27,46{ }^{\circ} \mathrm{C}$ à la grande saison pluvieuse. Les mois de février, mars et avril plus chauds connaissent des journées chaudes $\left(31-33{ }^{\circ} \mathrm{C}\right)$ suivies des nuits fraîches $\left(24-26^{\circ} \mathrm{C}\right)$.

\section{Méthodologie}

L'approche méthodologique utilisée a consisté à la collecte des données historiques notamment les coupes sédimentologiques et palynologiques. Les coupes géologiques utilisées sont réalisées à partir de cinq (5) forages profonds de 26 à $42 \mathrm{~m}$ et douze (12) petits sondages dont les profondeurs varient de 2,70 à près de $10 \mathrm{~m}$ (Figures $1,2,3$ et 4). Les forages profonds notés AH-L, Ah-G et Hou ont été réalisés par la Direction de l'Hydraulique dans le cadre du projet «hydraulique villageoise, zones lacustres » et ont été décrits par Agassounon (2002). La chronologie pour ces dépôts a été basée sur la corrélation avec les coupes P-N (Oyédé, 1991) et Djonou (Laibi, 2011) datées au ${ }^{14} \mathrm{C}$ (Figure 3 et 4). Les petites carottes GA, AG, TO et $P$ non datées ont été étudiées par Oyédé (1991). La lithologie de la coupe YEV1 a été obtenue à partir de forages réalisés dans le cadre du projet «Dahomey Gap " (Ballouche et al., 2001) et les mesures chronologiques au ${ }^{14} \mathrm{C}$ ont été faites par Agassounon (2002). Les sondages GOHO (Goho), SOA (Sô-Ava), DO (Dogla-lago) ont été exécutés grâce à une coopération entre l'Université d'Angers, l'Université Nationale du Bénin et l'Université de Francfort dans le cadre d'une étude sur le «Dahomey Gap » (Agassounon, 2002). La carotte $G$ a été collectée des travaux de Agassounon (1998). Les coupes S3, S4, S10 et Kpa non datées, ont été réalisées dans le cadre des travaux de Adéchina (2013). Les coupes palynologiques réalisées dans le delta sont peu nombreuses et couvrent seulement la période des 7500 ans B.P. Deux coupes GO et SO (Figure 5,6) ont été finalement retenues des travaux de Tossou (2002).

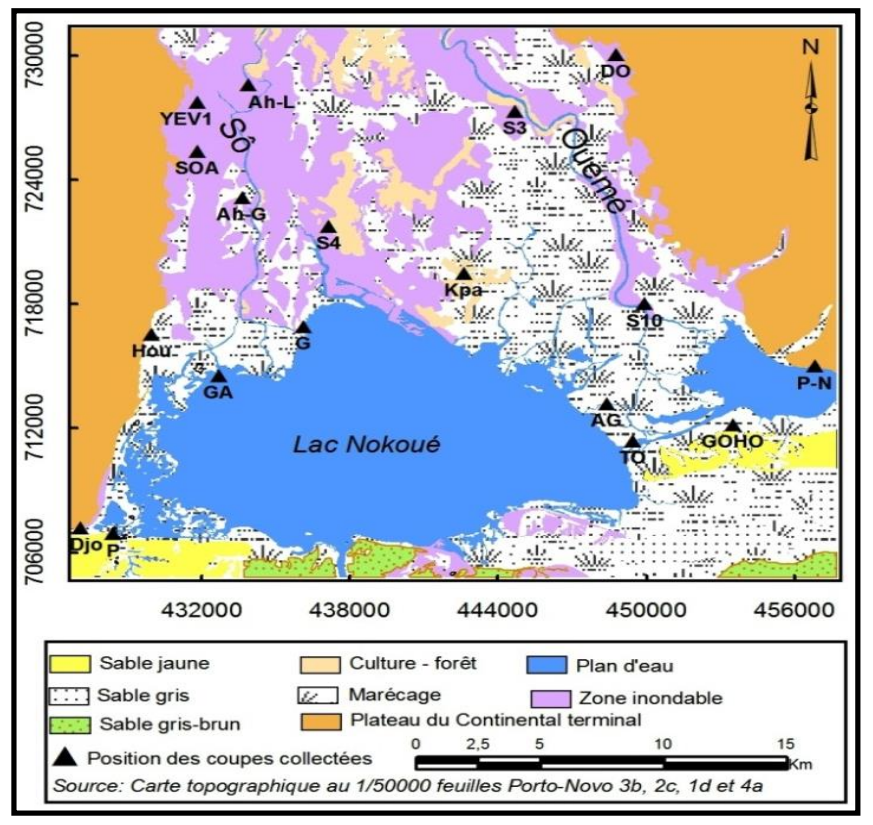

Figure 1 : Localisation du delta de l'Ouémé, des coupes géologiques et palynologiques (Agassounon, 2002). 
R. M. A. ADECHINA et al. / Int. J. Biol. Chem. Sci. 12(2): 1068-1085, 2018

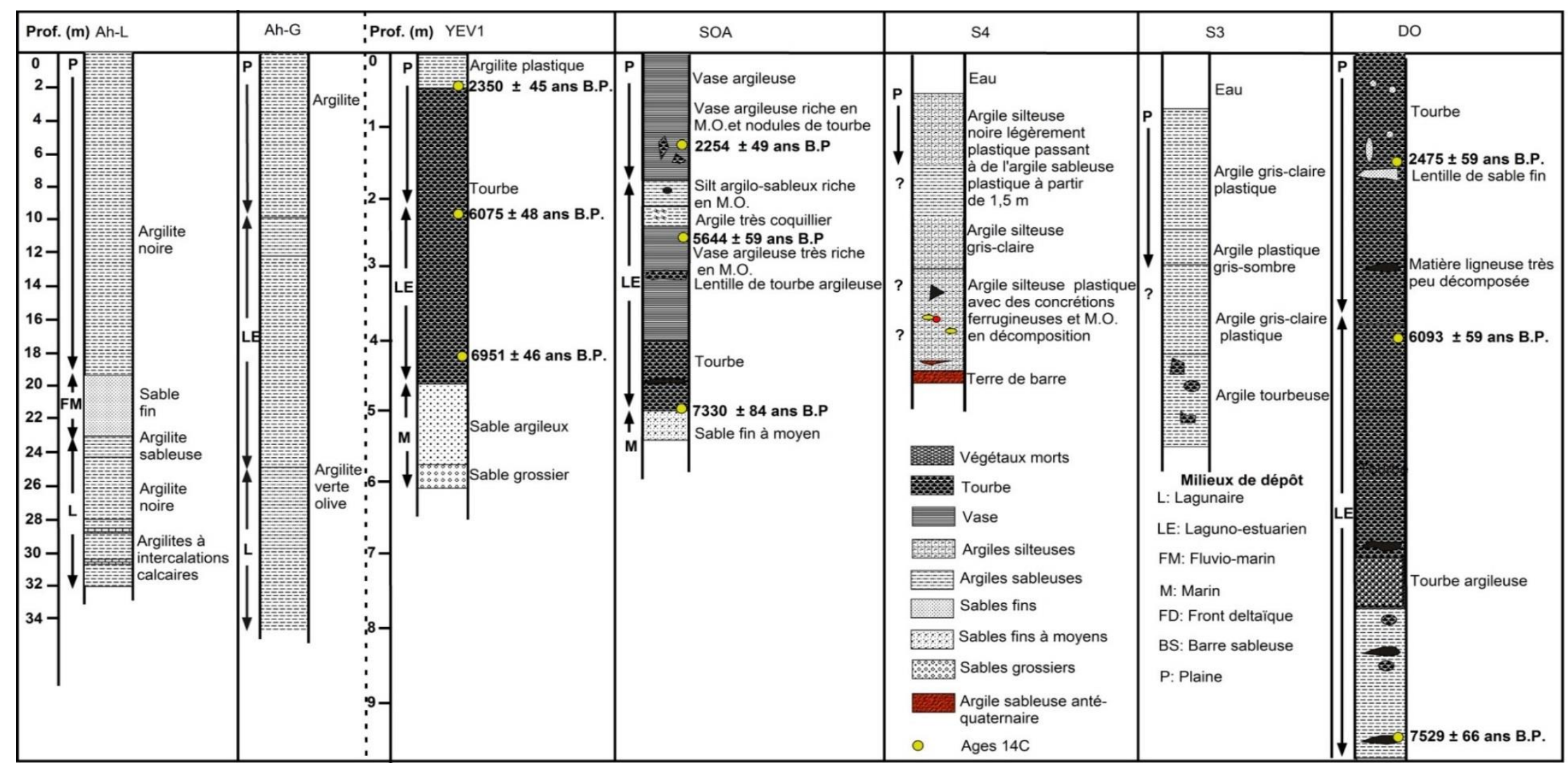

Figure 2: Coupes lithostratigraphiques des sondages Ah-L, Ah-G, YEV 1, SOA, S4, S3 et DO (Ballouche et al., 2001 ; Agassounon, 2002 ). 
R. M. A. ADECHINA et al. / Int. J. Biol. Chem. Sci. 12(2): 1068-1085, 2018

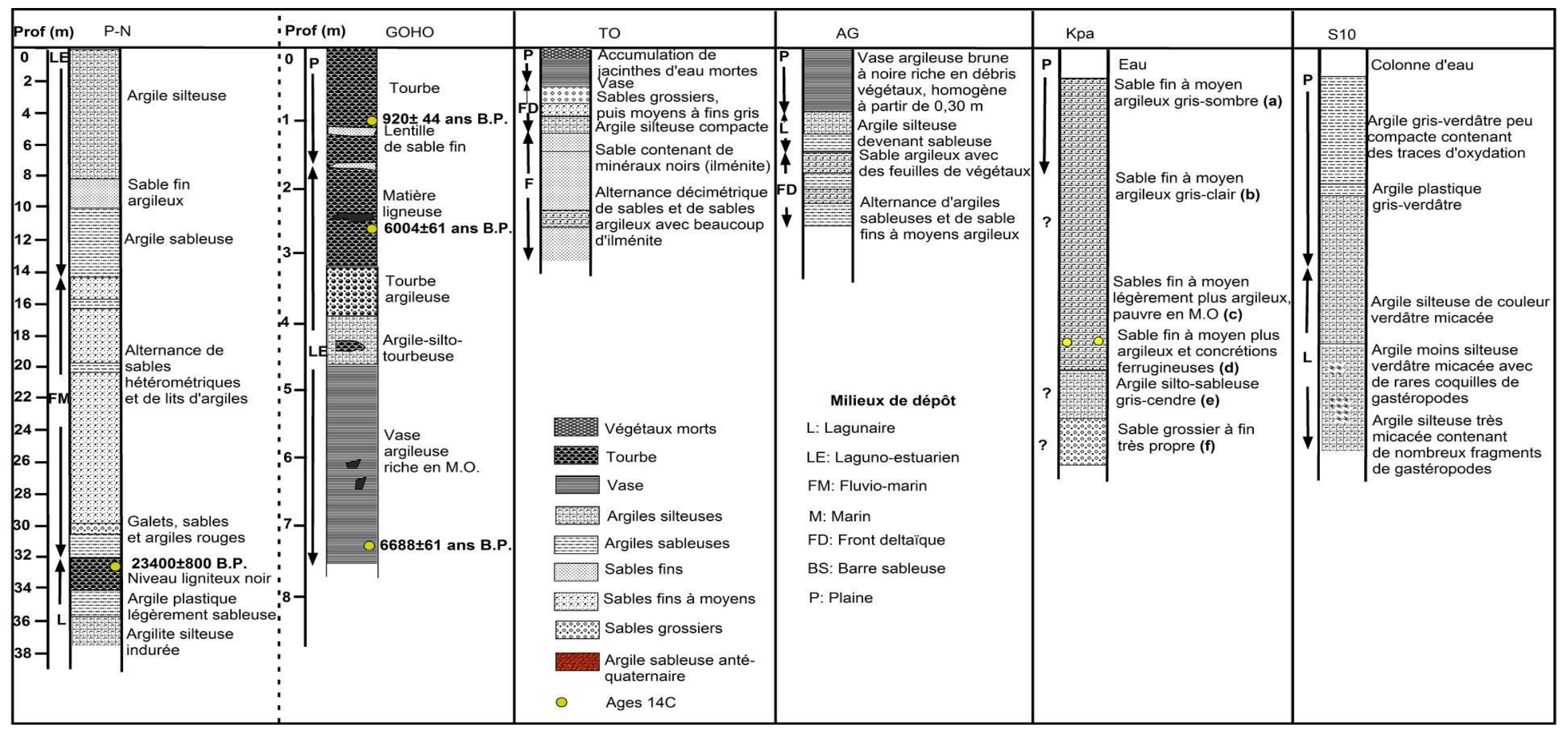

Figure 3 : Coupes lithostratigraphiques des sondages P-N, GOHO, TO, AG, Kpa et S10 (Oyédé, 1991, Adéchina, 2013). 


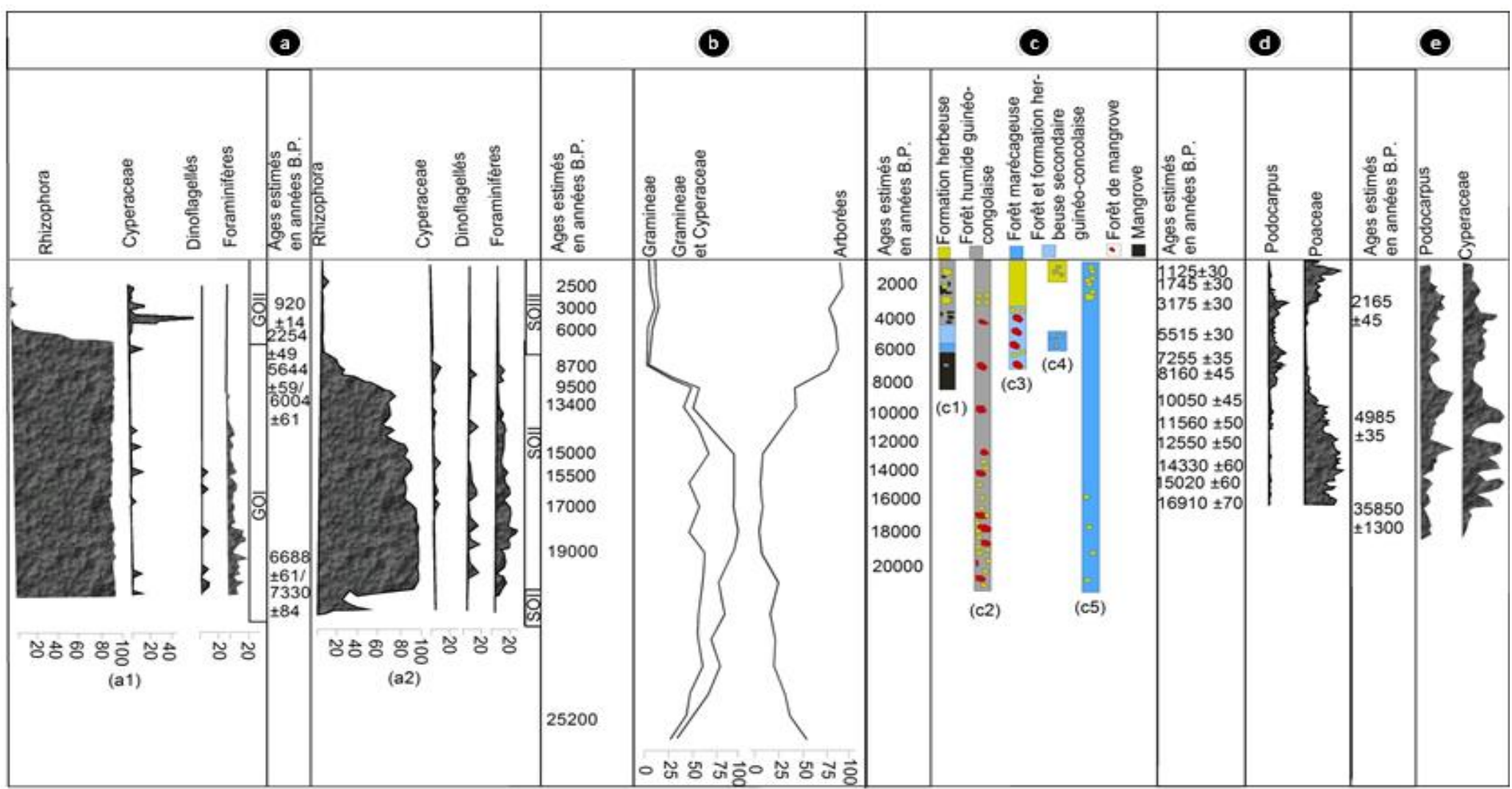

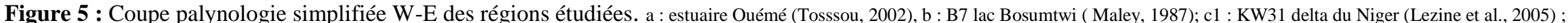
c2 : Barombi-Mbo (Assi-Kaudjhis, 2011) ; c3 : M’balang (Vincens et al., 2010) ; c4 : Sinda (Vincens, et al., 1998); c5: Ngamakala (Elenga et al., 1998) ; (d) Bambili (Assi-Kaudjhis, 2011) ; (e) Kivu (Kabonyi et al., 2015) 


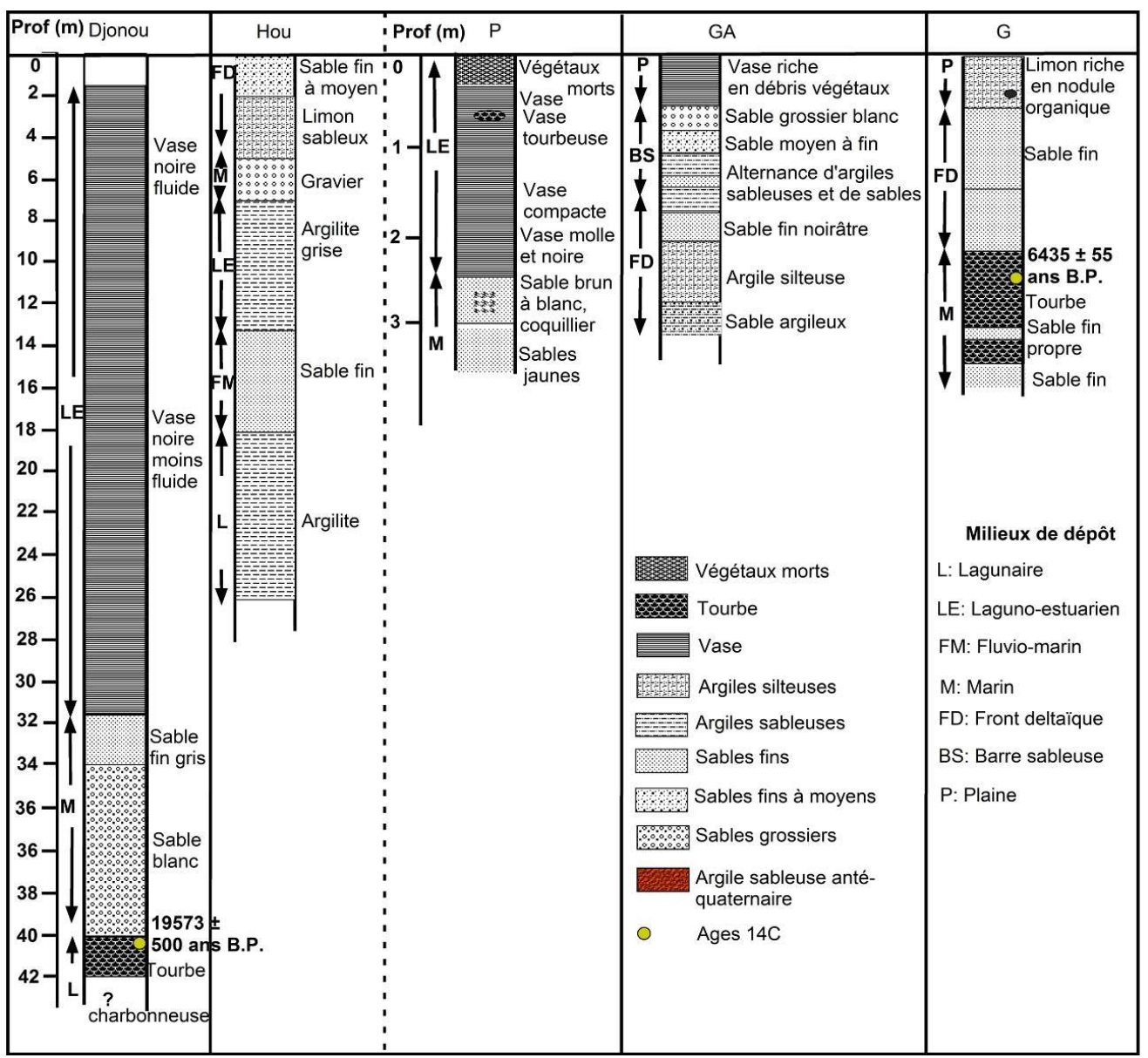

Figure 4 : Coupes lithostratigraphiques des sondages Djonou, Hou, P, GA et G (Lang et al., 1988 ; Oyédé, 1991; Laibi, 2011).

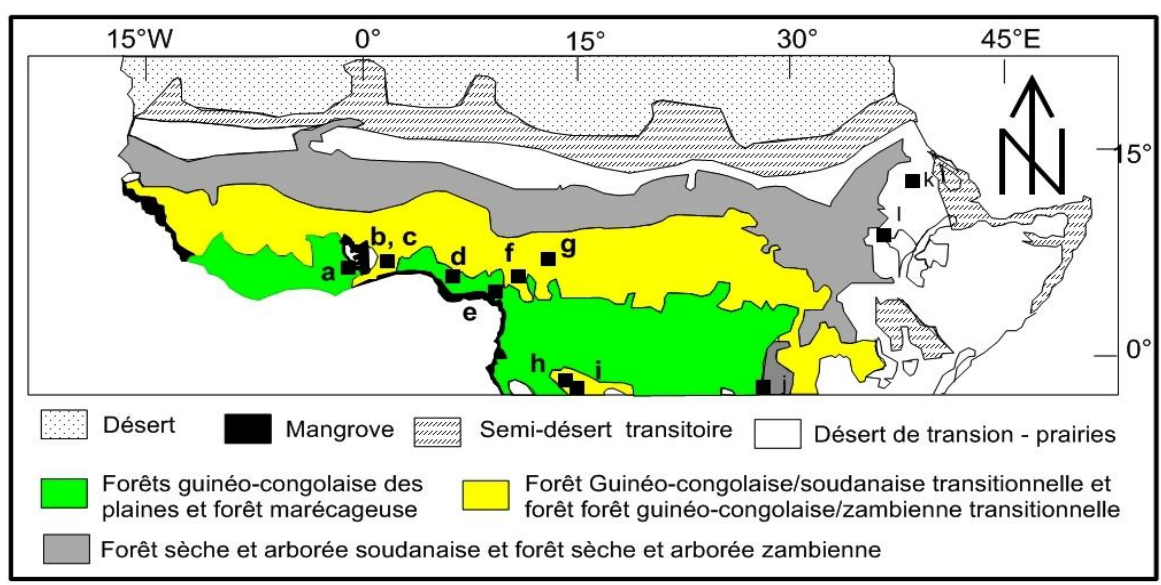

Figure 6: Localisation des coupes palynologiques et limnologiques avec le contexte végétatif. a. Bosumtwi ; b, c. SOA et Goho ; d. delta du Niger, e. Barombi M'bo, f. Bambili ; g. M’balang ; h. Sinda ; i. Ngamakala ; j. Kivu ; k. Abhe ; 1. Ziwah (Elenga et al., 2001 ; Ballouche et al., 2001 ; Tossou, 2002 ; Nyssen et al., 2003 ; Lezine et al., 2005 ; Vincens et al., 2010 ; Assi-Kaudjhis, 2011; Damnati et al., 2014 ; Kabonyi et al., 2015). 


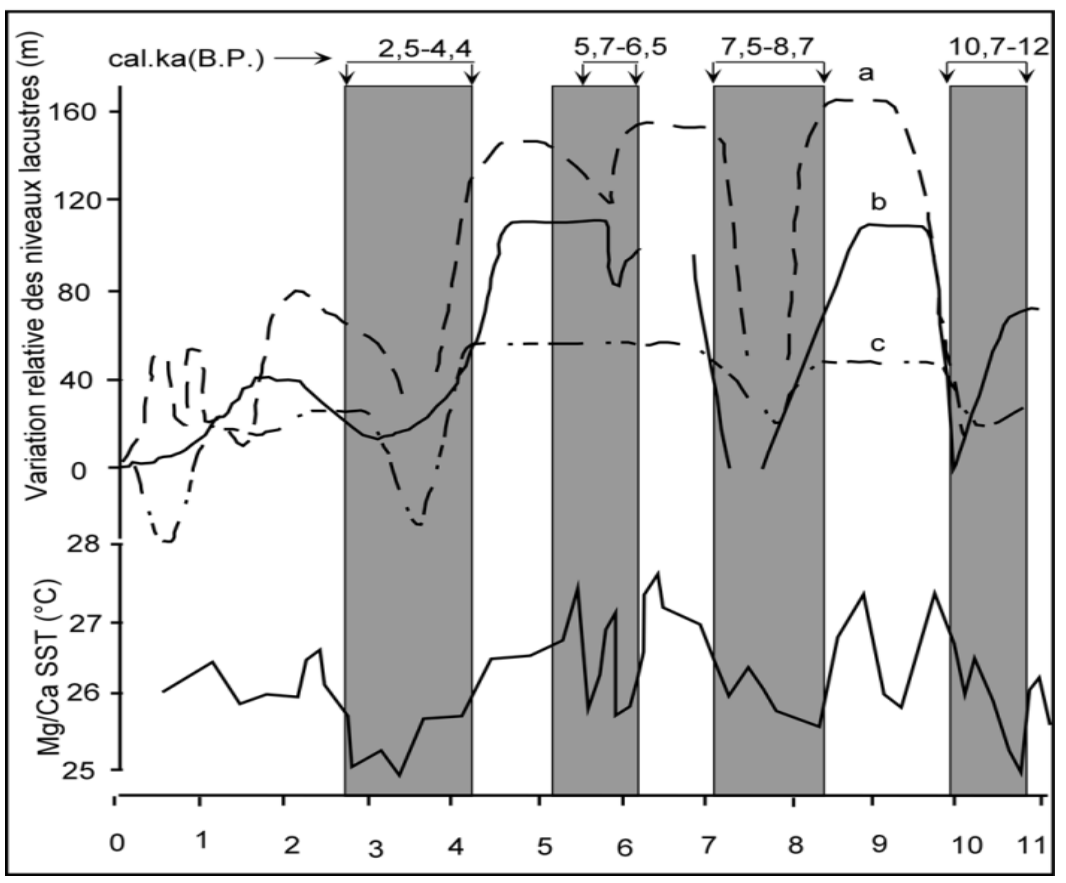

Figure 7: Corrélation entre les températures de surface océaniques holocènes (Sea-Surface Temperature, SST) et les fluctuations des niveaux lacustres (Weldeab et al., 2014). (a) lac Abhè et (b) complexe Ziway-Shala (Nyssen et al., 2003), (c) lac Bosumtwi (Maley, 1987). La bande grise montre la corrélation entre la baisse des SST et les bas niveaux lacustres.

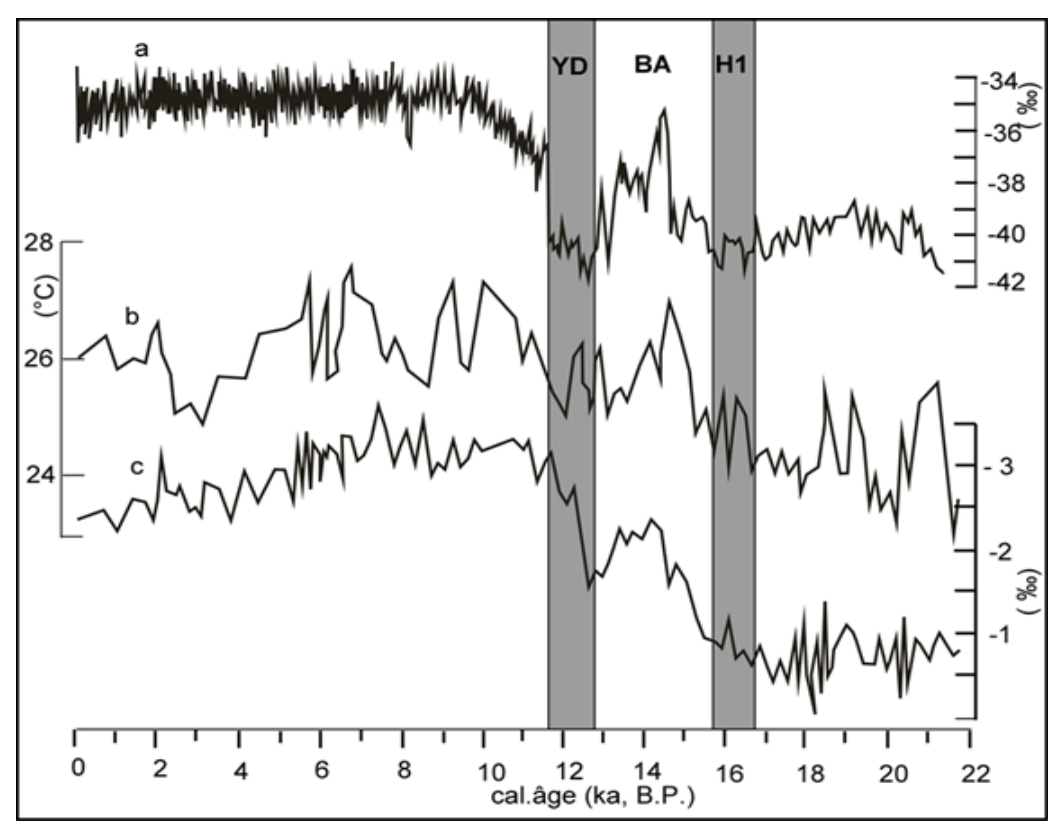

Figure 8 : (a) $\delta^{18} \mathrm{O}$ des calottes du GISP2 (Greenland Ice She et Project 2); (b) SST estimées à partir du Mg / Ca Globigerinoides ruber dans la carotte Geo B4905-4 au large du Cameroun ; (c) $\delta^{18} \mathrm{O}$ des G. ruber de Geo B4905-4 (Weldeab et al., 2014). 


\section{RESULTATS}

\section{Description des coupes géologiques}

Coupe Ah-L: E 2०24'10" ; N $6^{\circ} 35^{\prime} 30^{\prime \prime}$. Elle est réalisée dans la localité de Ahomey-Lokpo sur la rive Est de la rivière Sô, sur une profondeur de $154,5 \mathrm{~m}$. La présente étude a été focalisée sur les trentedeux (32) premiers mètres. Les dépôts sont essentiellement constitués d'argilites qui s'alternent avec des intercalations calcaires entre 28-32 m. Une intercalation de sables fins est notée entre 19,5-23 m (Figure 2).

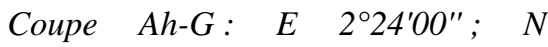
$6^{\circ} 32^{\prime} 30^{\prime \prime}$. Elle est située au Sud de Ah-L dans la localité de Ahomey-Glon et a une profondeur de $120 \mathrm{~m}$. La lithologie des 35 premiers mètres décrits est constituée d'argilites devenant sableuse entre 10-12 m puis $25-30 \mathrm{~m}$ (Figure 2).

Coupe YEV1: E2 $233^{\prime} 43^{\prime \prime}$; $N 6^{\circ} 29^{\prime} 43^{\prime \prime}$. D'une profondeur d'environ 5,60 m, elle est située dans la localité de Yévié. Sa lithologie est constituée de faciès sableux, grossier à la base et limoneux entre 4,55-5,50 m. Un épais recouvrement de tourbe est identifié vers le sommet (entre 0,45-4,6 m). Le niveau supérieur est constitué d'argilite plastique (Figure 2).

Coupe SOA : N 2०23'00', E 6 $33^{\circ} 43^{\prime}$ '. D'une profondeur de $5,20 \mathrm{~m}$, elle est située du côté de la rivière Sô dans la localité de SôAva. La lithologie du niveau inférieur est constituée de sable fin à moyen aux dépens duquel s'est accumulé $1 \mathrm{~m}$ de tourbe. Les niveaux supérieurs sont argilo-vaseux riches en débris de tourbe et en matières organiques (Figure 2).

Coupe DO : N 2'32'13', E 6 $6^{\circ} 36^{\prime} 16^{\prime}$. Elle est située dans le village de Dogla-lago en bordure d'une dépression marécageuse près du plateau de de Sakété. La sédimentation du bas est représentée par des argilites riches en MO (3-7,30 m) au-dessus du quelle s'est accumulée une épaisse couche de tourbe avec des lentilles sableuse entre 12,85 m (Figure 2).
Coupe $\quad$ S3: $\quad E 2^{\circ} 29^{\prime} 59.874$; $N 6^{\circ} 34^{\prime} 46.65^{\prime \prime}$. Elle est située au Nord-Ouest de la localité de Kessoumou en bordure du fleuve Ouémé. Les faciès reconnus sont formés d'argilite plastique contenant des galets de tourbe à la base $(4-5,5 \mathrm{~m})$ (Figure 2).

Coupe S4 : E437170 ; N721740. Elle est située dans la localité de Gbéssou et a une profondeur de $4,40 \mathrm{~m}$. Sa lithologique est essentiellement constituée d'argile silteuse sus-jacente aux formations argilo-sableuses rougeâtres de la "Terre de barre"(Figure 2).

Coupe S10: E449972; N718003.

Elle est positionnée au Sud de la localité de Gbodjè et est essentiellement constitué $\mathrm{d}$ 'argiles très coquillières de gastéropode vers la base (Figure 3).

Coupe : $\quad P-N$ : E2³6’35,7', ; $N 6^{\circ} 28^{\prime} 5,7^{\prime \prime}$. Elle est positionnée dans la zone de contact entre le domaine margino-littoral et le plateau de Sakété et a une profondeur de $39,6 \mathrm{~m}$. Sur les argilites silteuses et sableuses, repose une couche ligniteuse datée de $23400 \pm 800$ ans B.P. (Lang et Paradis, 1982 ; Oyédé, 1991). Le recouvrement de ce niveau de tourbe est constitué d'argiles rougeâtres surmontées de niveaux de sables. Ces sables sont d'abord grossiers et présentent ensuite une granulométrie fine à moyenne entre 30-14 $\mathrm{m}$ avec des alternances de lits d'argiles. Le niveau supérieur argilosableux et silteux est recoupé par 1,6 m de sable fin argileux.

$$
\text { Coupe } \quad A G: \quad E 2^{\circ} 32^{\prime} 1,15^{\prime \prime} \text {; }
$$
$N 6^{\circ} 27^{\prime} 6,65^{\prime \prime}$. Sa profondeur est de 2,70 m et est située en bordure du distributaire de l'Ouémé qui mène à Aguégué-Hlazoumè. Les niveaux inférieurs (entre 1,5-2,70 m) sont caractérisés par une alternance de sables fins à moyens argileux et d'argiles sableuses. Le faciès argileux sus-jacent est vaseux de $0,9 \mathrm{~m}$ jusqu'à début du sondage.

Coupe TO: E2 $32^{\prime} 35,313^{\prime \prime}$; $N 6^{\circ} 26^{\prime} 7,92$ '. Située sur la rive nord du chenal de Toché, les agencements de faciès 
sont constitués par une alternance décimétrique de sables et de sables argileux riches en ilménite de la base jusqu'à 1,3 m. Une granocroissance allant des argiles silteuse compacte au sable moyens à fins puis grossiers est notée jusqu'à $0,6 \mathrm{~m}$. Sur ces sables reposent de la vase aux dépens du quelle s'est accumulé la jacinthe d'eau morte (Figure 3).

Coupe $\quad \mathrm{GOHO}: \quad E 2^{\circ} 34,805^{\prime}$, $N 6^{\circ} 26,560$ '. Elle est positionnée dans la localité de Goho, au Sud du débouché du canal de Toché dans la lagune de Porto-Novo et a une profondeur de 7,30 m. La sédimentation est essentiellement argileuse de la base jusqu'à $3,9 \mathrm{~m}$. Ces argiles sont tourbeuses vers leur toit et sont surmontées de tourbe argileuse $(3,9-3,15 \mathrm{~m})$ et de tourbe franche (Figure 3 ).

Coupe Kpa: E2०28'52.1',; N6 30'31.34', Elle est positionnée à l'intérieur de la terre émergée aux dépens duquel sont développées des forêts claires de la localité de Kpaffè. Sur les sables grossiers à fins propres, il est noté un agencement de dépôts allant des argiles silto-sableuses $(5,4-$ $4,7)$ aux sables fins à moyens argileux. Le niveau sableux entre 4,4-5,4 m renferme des concrétions ferrugineuses.

Coupe Hou : E 2०21'20" ; N $6^{\circ} 28^{\prime} 55,194^{\prime}$. Elle est réalisée dans la localité de Houekomè au Nord-Ouest du lac Nokoué notamment en bordure du plateau d'Allada et sa profondeur totale est de $58 \mathrm{~m}$. Les vingtsix (26) premiers mètres décrits montrent une lithologie très hétérogène. La séquence sédimentaire est globalement négative constituée d'argilite $(7-26 \mathrm{~m})$ intercalée de sable fin. Ces faciès sont recouverts de graviers et de sables fins à moyens (Figure 4).

$$
\text { Djonou : } \quad \text { E2²0’26,76', } \quad \text {; }
$$
$N 6^{\circ} 23^{\prime} 50,386^{\prime \prime}$. Elle est située au Nord du cordon de sable jaune dans le secteur de la lagune Djonou. Au-dessus d'un niveau inférieur de tourbe charboneuse (44-42 m) daté de $19573 \pm 500$ ans B.P., repose une séquence positive constituée de sable grossier à la base et de la vase vers le sommet (Figure 4).

Coupe GA: E 2०23'30', ; N $6^{\circ} 27^{\prime} 50^{\prime \prime}$. Elle est située près du débouché de la rivière Sô dans le lac Nokoué. D'une profondeur de $3,20 \mathrm{~m}$, sa lithologie montre une alternance de bancs sableux et argileux (Figure 4).

Coupe P: E 2०21'99,39', ; N $6^{\circ} 23$ '41,14', D'une profondeur d'environ $3,80 \mathrm{~m}$, elle est positionnée dans les vasières de Godomey au Sud-Ouest du lac Nokoué. Sur les sables, brun à blanc coquillier entre 2,40-3 m, jaunâtre à la base du sondage, reposent $2 \mathrm{~m}$ de vases. Ces vases sont tourbeuses avec des débris de végétaux reconnaissables vers leur toit $(1,10-0,40 \mathrm{~m})$ et l'ensemble est recouvert d'une accumulation de végétaux (Figure 4).

$$
\text { Coupe G: E2²5'11.9994', ; }
$$
$6^{\circ} 29 ’ 24$ '. Elle est positionnée dans le village de Vèki au Nord d'un chenal distributaire de la Sô et est profond de 3,6 m. Sur le niveau inférieur de sable fin s'est accumulé $1,2 \mathrm{~m}$ de tourbe montrant une mince intercalation de sable fin blancs autour de 3,1-3,3 m. Les niveaux supérieurs sont globalement sableuses avec un toit limoneux riche en M.O. (Figure 4).

\section{Contexte géologique de dépôts des séquences sédimentaires \\ Première séquence sédimentaire d'âge ogolien}

$\checkmark \quad$ Description : la lithologie de la base des coupes les plus profondes notamment Ah-L (23-32 m), Ah-G (25-35 m), P-N (34$37,5 \mathrm{~m}$ ) et Hou (18-26 m) (Figure 2, 3 et 4), est constituée par des argilites silto-sableuses noires parfois en alternance avec des niveaux calcaires. Ces sédiments sont déposés dans des conditions hydrodynamiques calmes, caractéristiques d'un milieu lagunaire. Aux dépens de ces faciès déposés dans la lagune 
de Porto-Novo, sont accumulés des tourbes datées de $23400 \pm 800$ ans B.P. (Oyédé, 1991) (Figure 3). Un âge proche de $19573 \pm$ 500 ans B.P. est reconnu pour des tourbes accumulées à la base de la coupe exécutée dans la dépression Djonou (Figure 4).

Contexte de dépôt : les âges évalués sur les niveaux tourbeux attestent que ces dépôts lagunaires ont lieu pendant la période régressive ogolienne. Durant cette période et dans les dépressions lagunaires, il s'était entretenu un environnement marécageux favorable à la prolifération des végétaux lesquels représentent les niveaux tourbeux. Pendant cette régression à l'origine du creusement des vallées, sont accumulés dans les chenaux fluviatiles et les plaines d'inondation aggradantes, des sables grossiers blancs, des graviers quartzeux, des sables argileux contenant des poches tourbeuses (Agassounon, 2002). L'ensemble de ces dépôts lagunaires et fluviatiles représente la séquence régressive ogolienne.

Deuxième séquence sédimentaire d'âge holocène inférieur - moyen

$\checkmark$ Description : sur les faciès lagunaires est accumulée une épaisse couche de sables quartzeux fins à grossiers et coquilliers, de sables à intercalations argileuses. Cet agencement lithologique est reconnu dans les sondages comme Ah-L (19,5-23 m), P-N (14,5-32,1 m), Djonou (32,5-40 m) et Hou (13-18 m). Selon Oyédé (1991), les faciès sableux ont un caractère hétérométrique reflétant une origine proche à partir des matériaux anté-quaternaires arrachés des plateaux du "Continental terminal". Ces faciès sableuses sont également identifiés à la base des petites coupes notamment YEV 1 (4,55-5,60 m), SOA (4,9-5,2), P (3 - 2,40 m), G (3,40-3,60 $\mathrm{m}$ ), (Figure 2, 3 et 4 ). Les tourbes qui leurs sont sus-jacentes sont datées respectivement de $6951 \pm 46$ ans B.P. à $6075 \pm 48$ B.P. dans la coupe YEV 1, de $7330 \pm 84$ dans SOA.
Contexte de dépôt: ces faciès sableux représentent un placage transgressif pendant la dernière transgression holocène amorcée autour de 7500 ans B.P. Plusieurs auteurs à la suite de Guilcher (1959) avaient daté l'amorce de la dite transgression entre 6000-5000 ans B.P. Selon Oyédé (1991) les légères fluctuations qui ont été associées à cette transgression, ont été soldées par la formation de la première génération de cordons de sables jaunes (autour de 5500 ans B.P.). En arrière des cordons littoraux formés, il a été déroulé une importante sédimentation fine et le développement d'un système de ria à mangrove. Ainsi, dans un environnement progressivement lagunoestuaire ont été accumulées des argiles siltosableuses et des tourbes des localités de Ahomey-Glon (Ah-G 10-25 m), Dogla-lago (DO 8,10-9,90 m), Hou (7-13 m) et probablement celles de Gbéssou (S4 1,5-3 m) et du Nord-Ouest de Kessoumou (S3 2,5 - jusqu'à la base). Les datations faites sur les tourbes de la coupe DO ont attesté un âge de $7529 \pm 66$ à $6093 \pm 59$ ans B.P. (Figure 2 et 4). Ces faciès laguno-estuaire sont également identifiés en bordure les dépressions marécageuses (exemple P-N 10-14,2 m) et sont associés à d'épaisse couche de vases riches en matières organiques (M.O.), aux tourbes argileuses et aux tourbes dans les coupes de Goho $(6688 \pm 61$ à $6004 \pm 61$ ans B.P.), Djonou et Godomey. L'ensemble de ces dépôts fluvio-marins, laguno-estuariens correspond à la séquence liée à la transgression nouakchottienne.

\section{Troisième séquence sédimentaire d'âge holocène supérieur \\ $\checkmark$ Description : sur les argilites} laguno-estuariens reconnues entre 7 et $13 \mathrm{~m}$ dans la coupe Hou, sont accumulés des graviers (Hou 5-7 m). Cette succession lithologique avait été signalée dans la localité de Ganvié (Agassounon, 2002). Selon Oyédé (1991), ces dépôts sont caractérisés par un granoclassement négatif de sables grossiers à 
fins avec de minces laminations siltoargileuses et sont fortement coquilliers.

Contexte de dépôt : le dépôt de ces sédiments est fait suite à une légère baisse du niveau marin qui est maintenu supérieur à l'actuel. Ces dépôts ont été effectués pendant la phase régressive de l'Holocène supérieure. Une telle séquence négativement granoclassées caractéristique de ces dépôts est également reconnue dans le secteur de Kpaffè, avec des sables fins à moyens gris claire, très argileux vers le bas et pauvre en MO (Kpa 3,40 - 4,40 m).

Quatrième séquence sédimentaire d'âge holocène supérieure

$\checkmark \quad$ Description : les niveaux supérieurs des coupes sont constitués d'argiles sableuses avec des éléments grossiers vers le sommet. C'est le cas des coupes TO (0,6-2,7 m), AG (1,2-2,7), G (0,6-2,2 m). Ces sédiments sont couronnés par des argiles silteuses, des tourbes et des boues contenant des végétaux peu décomposés.

$\checkmark \quad$ Contexte de dépôt : ces dépôts subactuels à actuels ont lieu dans un environnement fluvio-lagunaire avec des incursions périodiques de la marée. Ils sont déposés dans un contexte de la plaine deltaïque et du front deltaïque. Les dépôts du front deltaïque sont représentés par des barres sableuses de chenaux et d'embouchure et sont agencés suivant une séquence négative de types «coarsening upward» (Oyédé, 1991). Ils sont recouverts par ceux de la plaine deltaïque constitués de boues avec des végétaux peu décomposés. La corrélation entre les alternances d'argiles et de sables fins à moyens du sondage AG $(1,8-2,7 \mathrm{~m})$ interprétées comme ayant lieu en bordure lagunaire avec les argiles micacées contenant des fragments de coquilles du Sud de Gbodjè (S10 4-6,5 m) met en évidence une progradation des dépôts de la plaine en direction du complexe lagunaire du lac Nokoué-lagune de Porto-Novo. Des sondages plus profonds des secteurs du Sud de Gbodjè
(S10) et du Nord du canal Toché permettrait d'identifier les séquences liées à la transgression holocène et sous-jacentes aux dépôts lagunaires. Par ricochet, des corrélations pourraient être établies avec les dépôts liées à la dite transgression des dépressions lagunaires de Porto-Novo et de Goho.

\section{Palynologie}

Les travaux sur la palynologie de l'estuaire sont peu nombreux et limités à la période des 7500 ans B.P. L'analyse palynologique concerne deux coupes GO (ou Goho) et SO (SOA) dont le contexte géologique a été décrit plus haut. Ballouche et al. (2001) et Tossou (2002) ont défini une première zone pollinique à Rhizophora (50$95 \%$ ) entre 7300 et 2500 ans B.P. Dans les palynomorphes identifiés, il est noté une proportion importante de dinoflagellés contrairement au Cyperaceae qui ont régressé. Entre 2500 et 2000 ans B.P., les Rhizophora qui régressaient ont fini par disparaitre (Figure 5a.). Selon Tossou (2002), le développement des Rhizophora entre 7300 et 2500 ans B.P. a été effectué à proximité des forêts marécageuses et semi-décidues. Leur disparition était associée à réduction de l'espace forestier, au défrichement et à l'installation de la végétation anthropique.

\section{DISCUSSION}

Le contexte paléoclimatique de dépôt des sédiments au cours des 20000 ans B.P. est situé à l'échelle intertropicale africaine et à l'échelle globale (Figures 5 et 6 ). Les indicateurs du paléoclimat régional sont la palynologie, la limnologie et les "Sea-Surface Temperature, SST". Les données palynologiques sont celles des régions de Barombi-Mbo, Bambili (Assi-Kaudjhis, 2011) et M'balang (Vincens et al., 2010) au Cameroun; de Bosumtwi au Ghana (Maley, 1987) ; de Kivu dans les marais de Cishaka en RDC (Kabonyi et al., 2015); de Sinda au 
Congo (Vincens et al., 1998), de Ngamakala au Sud Congo (Elenga et al., 1994); du delta du Niger au Nigéria (Lezine et al., 2005). Les courbes limnologiques sont celles : de Bosumtwi (Maley, 1987); du lac Abhe et du complexe Ziway-Shala (Nyssen et al., 2003).

\section{Palynologie et paléoclimat des 20000 ans} B.P. en Afrique intertropicale

Dans la région du lac Bosumtwi (Ghana) située à l'Ouest de l'Ouémé, les forêts ont régressé énormément entre 19000 et 15000 ans B.P. au profit des Graminées et des Cyperacées (Figure 5.b). Cette condition aride a été modérée dans la région de Barombi-Mbo au Cameroun où ont été identifiées des zones de refuge forestier (Figure 5.c2). Par analogie aux régressions forestières des régions centrales et orientales de l'Afrique intertropicale, Van Zinderen Bakker (1967) pense que le territoire territoire bénino-togolais appelé «Dahomey Gap » résulte d'un important rétrécissement de forêts denses pendant les périodes glaciaires. Vers 13400 ans B.P., une tendance humide a été enregistrée à Bosumtwi. Mais, l'environnement de Barombi-Mbo a été trop tôt recolonisé par des éléments afromontagnards et guinéo-congolais dès 15000 14080 ans B.P. (Assi-Kaudjhis, 2011 ; Figure 5.c2). Entre 12000-10000 ans B.P., Maley (1987) et Kabonyi et al. (2015) ont remarqué une interruption aride favorable à une modeste progression des Poaceae, des Gramineae et des Cyperaceae (Figure 5b). Cette aridité est suivie d'une période humide favorable à l'extension des taxons forestiers de Bambili (Cameroun) entre 10300- 4600 cal B.P. (Figure 5.d) et au large du delta du Niger entre 9700 - 8500 B.P. (Figure 5.c1). Dans l'estuaire de l'Ouémé, cette phase humide coïncide avec le développement des Rhizophora lesquels ont disparu dès l'Holocène récent suite à un déficit hydrique (Tossou, 2002). Dans ce contexte climatique, des apparitions brutales de Podocarpus entre
4300 cal B.P. et 3400 cal B.P. ont été signalées à Bambili. Il a été aussi remarqué un mixage de taxons herbacés de savane et ligneux de forêt vers 3000-2500 B.P. à Barombi Mbo et à M'balang au Caméroun (Figure 5.c1 et c3).

\section{Limnologie des 20000 ans B.P. en Afrique intertropicale}

Les reconstructions des niveaux lacustres dans le Sahara, l'Afrique occidentale et orientale ont attesté d'un niveau bas ou intermédiaire entre 22000 et 12000 ans B.P. (Maley, 1987 ; Nyssen et al., 2003 ; Damnati, 2014). Cependant, une impulsion position a été notée autour de 11500 ans cal B.P. dans le système lacustre Ziway-Shala. D'autres, concomitamment dans les lacs Abhe et Bosumtwi ont été signalées entre 9500-8000, 7000-6000 et 5500- 4500 cal B.P. (Figure 7 a, b, c). Contrairement au lac Abhe et Ziway-Shala qui ont connu une baisse faiblement prononcée de 6500 à 5700 ans B.P., aucune modification remarquable du niveau du lac Botsumwi n'a été observée. Vers 4800- 4200 ans ${ }^{14} \mathrm{C}$ B.P., les lacs se sont retirés au bas niveau. Cependant, trois (3) pulsations positives ont été enregistrées autour de 30001500 ans, 1200 ans et 500 ans cal B.P. dans le lac Abhe. Celles du lac Bosumtwi furent entre 3000 - 2500 ans B.P. et autour de 1200 ans B.P.

\section{Paléotempérature des 20000 ans B.P. en Afrique intertropicale}

Les reconstitutions des températures disponibles pour le milieu continental au Ghana, au Cameroun et au Tchad ont permis d'estimer le refroidissement du dernier maximum glaciaire (23000-18000 ans B.P.) entre 5-10 ${ }^{\circ} \mathrm{C}$ par rapport à l'actuel (AssiKaudjhis, 2011. Clark et al. (2012) a suggéré $4{ }^{\circ} \mathrm{C}$ pour la grande partie de l'Afrique tandis que Truc et al. (2013) l'ont évalué à $6 \pm 2{ }^{\circ} \mathrm{C}$ dans les tropiques sud-africains. Dans le 
milieu marin, la moyenne des températures annuelles (SST) du golfe de Guinée a été d'environ $25 \quad{ }^{\circ} \mathrm{C}$ (Lezine, 1997). Ces températures auraient été liées à l'intensification des remontées d'eaux froides "upwellings". Les SST de l'Atlantique équatorial est au large du Cameroun ont attesté d'un léger réchauffement de 24,7 \pm $0,6{ }^{\circ} \mathrm{C}$ entre $17000-15500$ ans cal B.P. (Weldeab et al., 2014 ; Figure 8.b et c). Les SST de l'Atlantique équatorial Est ont ensuite décru entre 12000-10000 ans B.P. avant de remonter à près de $27{ }^{\circ} \mathrm{C}$ autour de 9700 ans B.P. Quelques interruptions froides ont été relevées entre $9700-9200 ; 8700$ 7500; $6600-6300$ et $6000-5700$ ans calendaire B.P. (cal B.P.). Celle de $8700 \pm$ 0,2 ans cal B.P. a été de $24,9^{\circ} \mathrm{C}$. La période de 7000 - 6000 ans B.P. correspond au maximum thermique de $27,5{ }^{\circ} \mathrm{C}$ centré sur 6700 ans cal B.P. dans l'Atlantique équatoriale Est (Figure 8.b et c). Des valeurs minimales de SST d'environ $25{ }^{\circ} \mathrm{C}$ ont été enregistrées entre 3500 et 2600 ans cal B.P. et d'autres moins prononcées entre 1700 1000 ans B.P. et autour de 500 ans cal B.P.

\section{Paléoclimat globale et contexte géologique des sédiments de l'estuaire de l'Ouémé}

$\mathrm{La}$ palynologie des régions intertropicales africaines confortent bien les enregistrements du «Greenland Ice Sheet Project 2 : GISP2) basés sur les variations du $\delta^{18} \mathrm{O}$ (Grootes et al., 1993). Le refroidissement du dernier maximum glaciaire (23000 - 18000 ans B.P.) est concomitant au stade de rétrécissement des forêts, au bas niveau marin et lacustre, à la baisse du $\delta^{18} \mathrm{O}$ et des SST. Les anomalies d'insolation en baisse vers les latitudes $10^{\circ} \mathrm{N}$ ont varié entre 10 et $15 \mathrm{~W} \cdot \mathrm{m}^{-2}$ (Berger et Loutre, 1991; Bubenshchikova et al., 2015). C'est dans ce contexte que s'est déposée la séquence de bas niveau marin ogolien dans l'estuaire de l'Ouémé. Dans la logique d'un déplacement de la ZCIT, Clark et al. (2012) pensent que des conditions arides ont prévalu au Nord de la ZCIT et des conditions plus humides au Sud dans les régions tropicales d'Afrique. Ainsi, une tendance chaude a été attestée par les SST moyennes et correspond aux évènements d'Heinrich 1 (17000 - 15500 ans cal B.P.) dans l'Hémisphère Nord (Penaud et al., 2016). Le retour à une condition humide après 15000 ans B.P. avec une nouvelle recolonisation forestière en Afrique intertropicale (Assi-Kaudjhis, 2011 ; Kabonyi et al., 2015) est contemporain au Bölling-Alleröd (15000-12500 ans B.P.; B/A, Figure 8). La palynologie et les SST de l'Atlantique équatorial Est (Cameroun) montrent une interruption aride entre 12000 10000 ans B.P. coïncidant avec le Younger Dryas (12800 - 11700 B.P.). Dans les hautes latitudes de l'hémisphère Nord, l'ensoleillement d'été a atteint son maximum vers 11000 ans B.P. (Debret, 2008). Cette phase a été accompagnée par le maximum d'ensoleillement à la latitude $10^{\circ} \mathrm{N}$, la hausse $\mathrm{du}$ niveau marin, les transgressions forestières en Afrique intertropicale. La péjoration climatique notée autour de 37002000 B.P. dans l'estuaire de l'Ouémé ou globalement dans l'Afrique intertropicale coïncide à un début du Néoglaciaire centré sur 3250 B.P. dans l'Hémisphère Nord (Van Vliet-Lanoë et al., 2014)

\section{Quelques lacunes et controverses relevées Contexte géologique de dépôt}

Des séquences sédimentaires évoquant des environnements lagunaires, fluvio-marins ont été reconnues dans la partie intérieure de l'estuaire de l'Ouémé notamment dans les localités de Kpaffè, de Gbodjè et des Aguégués. Leur corrélation avec celles identifiées dans l'estuaire de la Sô et dans les dépressions lagunes situées à la limite du domaine des plateaux et le domaine margino-littoral, est hypothétique en raison de l'insuffisance des données chronologiques et des sondages plus profonds dans ces secteurs intérieurs de l'estuaire de l'Ouémé. 


\section{Contexte paléoclimatique}

Des évènements séculaires à l'instar des refroidissements de 9700-9200 B.P. et 6500 - 5700 ans B.P. au large du Cameroun ne sont corrélés au bas niveau dans le lac Bosumtwi, contrairement aux lacs Abhe et Ziway-Shala qui ont connu une baisse faiblement prononcée de 6500 à 5700 ans B.P. A cela s'ajoute le refroidissement de 8200 ans B.P. dans l'Hémisphère Nord qui concorde avec la baisse des niveaux lacustres et le refroidissement de SST au large du Cameroun. Les études palynologiques et sédimentologiques de l'estuaire de l'Ouémé qui attestent une phase de transgression forestière et marine ne mettent pas en exergue ces interruptions froides d'échelle séculaire qui ont jalonné l'Holocène inférieur et moyen.

\section{Conclusion}

Les reconstructions paléoenvironnementales des 20000 ans B.P. du delta de l'Ouémé montrent une grande sensibilité de cet écosystème intertropical africain aux changements globaux d'ordre eustatique, paléoclimatique. Ces derniers ont été enregistrés par des faits tels que, les régressions/transgressions marines et forestières, les variations des apports fluviogéniques. Le refroidissement du dernier maximum glaciaire a été soldé par une régression des forêts et du niveau marin. Grâce à l'humidité du climat holocène, les forêts ont proliféré, l'estuaire de l'Ouémé a été transformé en ria. Il s'en est suivi vers 4200 ans B.P., une péjoration climatique au cours de laquelle sont amorcés les dépôts de la plaine deltaïque actuelle de l'Ouémé. Ces évènements suscitent d'importantes hypothèses à vérifier pour une meilleure compréhension de leur variation spatiale et temporelle. Les dites hypothèses sont liées à l'insuffisance des données chronologiques et des outils adéquats de reconstitution du paléoclimat. Les recherches devront être davantage orientées sur l'évolution du paléoclimat et du paléoenvironnement de dépôt de sédiment de l'Holocène à nos jours.

\section{CONFLIT D'INTERETS}

Les auteurs déclarent qu'il n'y a aucun conflit d'intérêts pour cet article.

\section{CONTRIBUTIONS DES AUTEURS}

ARMAA a assuré la collecte des coupes, l'analyse et l'interprétation des données. CNK a contribué à l'acquisition et à l'analyse des coupes palynologiques. CK et RAL ont contribué à l'acquisition et à l'analyse des coupes sédimentologiques. JBH a contribué à la digitalisation des coupes.

\section{REFERENCES}

Adéchina ARMA. 2013. Etude des dépôts de l'Holocène a l'actuel et spéciation chimique du plomb dans le bas-delta de la basse vallée de l'Ouéme-Sô. Mémoire de Master professionnel, Université d'Abomey-Calavi, p.108.

Agassounon LC. 1998. Le delta de la Sô (Bénin-Afrique de l'Ouest) : dynamique sédimentaire depuis 7000 ans B.P.; fluctuation du niveau marin dans le domaine margino littoral du Golfe de Guinée et relation avec les paléoenvironnements continentaux. Mémoire de DEA, Université de Dijon, Lyon I, Aix-Marseille I, Toulouse III, p.45.

Agassounon LC. 2002. Evolution pédosédimentaire du géosystème marginolittoral de l'Ouémé-Sô au cours de l'holocène (Bénin Afrique de l'ouest). Thèse de doctorat, Université de Bourgogne, Dijon, p.423.

Assi-Kaudjhis C. 2011. Dynamique des écosystèmes et biodiversité des montagnes du Cameroun au cours des derniers $20 \quad 000$ ans. Analyse palynologique d'une série sédimentaire du lac Bambili. Thèse de doctorat, Université de Versailles Saint Quentinen Yvelines, Université de Liège, p.173. 
Ballouche A, Akoegninou A, Neumann K, Salzmann U, Sowunmi MA. 2001. Le projet Dahomey Gap : une contribution à l'histoire de la végétation au SudBénin et au Sud-Ouest du Nigéria. Berichte des Sonderforschungsbereichs 268, Band 14, Frankfurt a.M., 2000: 237-251.

Berger A, Loutre MF. 1991. Insolation values for the climate of the last 10 million years, Quat. Sci. Rev., 10: 297317. DOI: http://dx.doi:10.1016/02773791(91)90033-Q.

Bubenshchikova N, Nürnberg D, Tiedemann R. 2015. Variations of Okhotsk Sea oxygen minimum zone: Comparison of foraminiferal and sedimentological records for latest MIS $12-11 \mathrm{c}$ and latest MIS 2-1. Mar. Microp., 121: 52-69. DOI: $\quad$ http://dx.doi.org/10.1016/ j.marmicro.2015.09.004

Clark UP, Shakun JD, Baker AP, Bartlein JP, Brewer S, Brook E, Carlson AE, Cheng H, Kaufman DS, Liu Z, Marchitto TM, Mix AC, Morrill C, Otto-Bliesner BL, Pahnke K, Russell JM, Whitlock C, Adkins JF, Blois JL, Clark J, Colman SM, Curry WB, Flower BP, He F, Johnson TC, Lynch-Stieglitz J, Markgraf V, McManus J, Mitrovica JX, Moreno PI, Williams JW. 2012. Global climate evolution during the last deglaciation. PNAS, 109(19): E 11341142. DOI:10.1073/pnas.1116619109//DCSupplemental.

Damnati B, Khadija KA, Benjilani H, El Afia M, El Khoudri K. 2014. Lake Level Variations for the Last 30,000 Yr B.P: New Palaeoclimatic Synthesis in Northern Hemisphere of Africa. ARPN J. Sci. Tech., 4(12): 1-8.

Debret M. 2008. Caractérisation de la variabilité climatique Holocène à partir de séries continentales, marines et glaciaires. Thèse de doctorat de l'Université Joseph Fourier, Grenoble 1, p308.
Elenga H, Schwartz D, Vincens A. 1998. Pollen evidence of late Quaternary vegetation and inferred climate changes in Congo, Palaeog., Palaeoclim., Palaeoeco., 109: 345-356.

Elenga H, Vincens A, Schwartz D, Fabing A, Bertaux J, Wirrmann D, Martin L, Servant M. 2001. Le marais estuarien de la Songolo (Sud Congo) à l'Holocène moyen et récent. Bull. Soc. Géol. France, 172(3): 359-366.

Green V, Bueno Watts N, Wegner K, Thompson M, Johnson A, Baptista AM. 2013. Coastal Margin Science and Education in the Era of Collaboratories. Currents, 28(3): 1-27.

Grootes PM, Stuiver M, White JWC, Johnsen S, Jouzel J. 1993. Comparison of oxygen isotope records from the GISP2 and GRIP Greenland ice cores. Nature, 366: 552-554. DOI : http://dx.doi: 10.1038/366552a0.

Guilcher A. 1959. La région côtière du BasDahomey occidental, Bull. Instr. Fr. Afr. Noir. Sénégal, t. XVI, sér. B, 100: 551-556.

Guo H-D, Zhang L, Zhu L-W. 2015. 2015. Earth observation big data for climate change research. Adv. in Clim. Chan. Resea., 6: 108-117. DOI: dx.doi.org/10.1016/j.accre.2015.09.007.

Intergovernmental Panel on Climate Change, Climate Change. 2014. Synthesis Report. Contribution of Working Groups I, II and III to the Fifth Assessment Report of the Intergovernmental Panel on Climate Change, Ed. Core Writing Team, R.K. Pachauri and L.A. Meyer. IPCC, Geneva, Switzerland.

Kaki C, Laibi R, Oyédé LM. 2011. Evolution of beninese coastline from 1963 to 2005 : causes and consequences. Brit. J. of Env. \& Clim. Ch., 1(4): 216-231.

Kabonyi NC, Roche E, Gerrienne P. 2015. Paléoenvironnements et paléoclimats durant le Pléistocène supérieur et 
l'Holocène sur la dorsale occidentale du Kivu en République Démocratique du Congo. Europ. Scie J., 11(30): 1-26.

Kélome N, Kaki C, d'Almeida GF, Adéchina R. 2012. Dynamique sédimentaire actuelle et subactuelle de la zone deltaïque de la basse vallée de l'OuéméSô. Rev. CAMES, série a, Sci Méd., 13(2): 221-225.

Laibi RA. 2011. Evolution quaternaire et dynamique sédimentaire actuelle des cordons barrières de l'estuaire du Mono-Couffo dans le littoral du Bénin (Golfe de Guinée, Afrique de l'Ouest), Thèse de doctorat, UAC, Bénin/ ULCO, France, p.307.

Lang J, Paradis G, Oyédé LM. 1988. Le domaine margino-littoral du Bénin (Golfe de Guinée Afrique de l'Ouest) : âge holocène et mise en place des sables jaunes. J. Afri. Ear. Sci., 7(5-6): 829833.

Lang J, Paradis G. 1977. Un exemple d'environnement sédimentaire, biodétritique, non carbonaté marin et continental, Holocène, en climat intertropical : Le domaine marginolittoral du Bénin méridional (ex Dahomey). Rev. Géogr. Phys. Géol. Dyn., 2(19): 295-312.

Lezine AM, Duplessy JC, Cazet JP. 2005. West African monsoon variability during the last deglaciation and the Holocene: Evidence from fresh water algae, pollen and isotope data from core KW31, Gulf of Guinea. Palaeog Palaeoclim., Palaeoeco., 219: 225-237.

Lézine A-M. 1997. Evolution of the West African Mangrove During the Late Quaternary: A Review. Géo. Phy. Quat., 51(3): 405-414.

Maley J. 1987. Fragmentation de la Forêt dense humide africaine et extension des biotopes montagnards au Quaternaire récent: nouvelles données polliniques et chronologiques. Implications, paléoclimatiques, chronologiques et biogéographiques. Paleo. Afri., 18: 307334.

Mix AC, Bard E, Shneider R. 2001. Environmental processes of the ice age: land, oceans, glaciers (EPILOG). Quat. Sci. Rev., 20: 627- 657.

Nyssen J, Poesen J, Moeyersons J, Deckers J, Haile M, Lang A. 2003. Human impact on the environment in the Ethiopian and Eritrean highlands-a state of the art. Ear. Sci. Rev., 64(3): 273-320. DOI:10.1016/S0012-8252(03)00078-3.

Oyédé LM. 1991. Dynamique sédimentaire actuelle et messages enregistrés dans les séquences quaternaires et néogène $\mathrm{du}$ domaine margino-littoral du bénin (Afrique de l'ouest), Thèse de doctorat, Université de Bourgogne, Dijon, p.302.

Penaud A, Eynaud F, Voelker AH, Turon JL. 2016. Palaeohydrological changes over the last $50 \mathrm{ky}$ in the central Gulf of Cadiz: complex forcing mechanisms mixing multi-scale processes. Biogeosciences, 13: 5357-5377. DOI:10.5194/bg-13-5357-2016.

Tossou MG. 2002. Recherche palynologique sur la végétation holocène du SudBénin (Afrique de l'Ouest), Thèse de doctorat, Université de Lomé, p. 133.

Truc L, Chevalier M, Favier C, Cheddadi R, Meadows ME, Scott L, Carr AS, Smith GF, Chase BM. 2013. Quantification of climate change for the last 20,000 years from Wonderkrater, South Africa: implications for the long-term dynamics of the Intertropical Convergence Zone. Palaeog Palaeoclim., Palaeoeco. Accepted manuscript. DOI: 10.1016/j.palaeo.2013.06.024.

Van Vliet-Lanoë B, Penaud A, Hénaff A, Delacourt C, Fernane A, Goslin J, Hallégouët B, Le Cornec E. 2014. Middle- to late-Holocene storminess in Brittany (NW France): Part II - The chronology of events and climate forcing. The Holocene, 24(4): 434-453. 
Van Zinderen Bakker EM.1967. Upper Pleistocene and Holocene stratigraphy and ecology on the basis of vegetation changes in subsaharah Africa. In Background of Evolution in Africa, Bishop WW, Clark JD (eds). University of Chicago Press: Chicago; 125-147.

Vincens A, Buchet G, Servant M, ECOFIT Mbalang collaborators. 2010. Vegetation response to the African Humid Period termination in central Cameroon $\left(7^{\circ} \mathrm{N}\right)$ - new pollen insight from Lake Mbalang. Clim. Past. Discuss., 5: 2577-2606.

Vincens A, Schwartz D, JBertaux J, Elenga H, De Namur C. 1998. Late Holocene Climatic Change in Western Equatorial
Africa Inferred from Pollen from lake Sinnda, Southern Congo. Quat. Res., 50: 34 - 45.

Weldeab S, Lea DW, Oberhänsl H, Schneider RR. 2014. Links between southwestern tropical Indian Ocean SST and precipitation over southeastern Africa over the last 17 kyr. Palaeog. Palaeocl. Palaeoeco, 410: 200-212. dx.doi.org/10.1016/j.palaeo.2014.06.00 1

Zannou AB, Vodounou KJB. 2011. Evolution des crues annuelles liées aux changements climatiques et adaptation des populations de la basse vallée de l'Ouémé. Mélange Mac, Université d'Abomey-Calavi, Bénin, p16. 